\title{
Inconsistent centralised versus non- centralised ischaemic stroke aetiology
}

\author{
Yue Suo (D) ,1,2 Jing Jing, ${ }^{1,2}$ Xia Meng, ${ }^{1,2}$ Zixiao Li (D) , ${ }^{1,2}$ Yuesong Pan (D) , ${ }^{1,2}$ \\ Yong Jiang, ${ }^{1,2}$ Xiaomeng Yang, ${ }^{1,2}$ Huan Liu, ${ }^{1,2}$ Hongyi Yan, ${ }^{1,2}$ Liping Liu (D) ,,2 \\ Xingquan Zhao, ${ }^{1,2}$ Yilong Wang, ${ }^{1,2} \mathrm{Hao}$ Li (D) , ${ }^{1,2}$ Yongjun Wang (D) ${ }^{1,2}$
}

\begin{abstract}
To cite: Suo Y, Jing J, Meng X, et al. Inconsistent centralised versus non-centralised ischaemic stroke aetiology. Stroke \& Vascular Neurology 2020;5: e000576. doi:10.1136/ svn-2020-000576
\end{abstract}

- Additional material is published online only. To view, please visit the journal online (http://dx.doi.org/10.1136/svn2020-000576).

Received 10 August 2020 Revised 3 October 2020 Accepted 7 October 2020 Published Online First 5 November 2020

\section{Check for updates}

(C) Author(s) (or their employer(s)) 2020. Re-use permitted under CC BY-NC. No commercial re-use. See rights and permissions. Published by BMJ.

${ }^{1}$ Department of Neurology, Beijing Tiantan Hospital, Beijing, China

${ }^{2}$ China National Clinical

Research Center for

Neurological Diseases, Beijing, China

Correspondence to Dr Yongjun Wang; yongjunwang@ncrcnd.org.cn

\section{ABSTRACT}

Background and purpose The Trial of Org 10172 in Acute Stroke Treatment (TOAST) system is the most widely used aetiological categorisation system in clinical practice and research. Limited studies have validated the accuracy of routine aetiological diagnosis of patients with ischaemic stroke according to the TOAST criteria when the reported subtype is assumed to be correct. We investigated the agreement between centralised and non-centralised (site-reported, at discharge) stroke subtypes in the Third China National Stroke Registry (CNSR-III), and analysed the influence of classification consistency on evaluation during hospitalisation and for secondary prevention strategy. Methods All patients with ischaemic stroke from the CNSR-III study with complete diffusion-weighted imaging data were included. We used multivariable Cox proportional-hazard regression models to evaluate the factors associated with consistency between centralised and non-centralised stroke subtypes. Sensitivity analyses were conducted of the subgroup of patients with complete information.

Results This study included 12180 patients (mean age, 62.3 years; and women, $31.4 \%$ ). Agreement between centralised and non-centralised subtype was the highest for the large-artery atherosclerosis subtype stroke (77.4\% of centralised patients), followed by the smallvessel occlusion subtype ( $40.6 \%$ of centralised patients). Agreements for cardioembolism and stroke of other determined aetiology subtypes were $38.7 \%$ and $12.2 \%$, respectively. Patient-level and hospital-level factors were associated with the inconsistency between centralised/ non-centralised aetiological subtyping. This inconsistency was related to differences in secondary prevention strategies. Only $15.3 \%$ of the newly diagnosed patients with cardioembolism underwent centralised subtyping with indications to receive oral anticoagulants at discharge. In comparison, $51.3 \%$ of the consistent cardioembolism group and $42.0 \%$ of the centrally reassigned cardioembolism group with anticoagulation indications were prescribed oral anticoagulants.

Conclusions Substantial inconsistency exists between centralised and non-centralised subtyping in China. Inaccurate aetiological subtyping could lead to inadequate secondary prevention, especially in patients with cardioembolic stroke.

\section{INTRODUCTION}

Stroke has become the second leading cause of death worldwide ${ }^{1}$ and the leading cause of mortality in China. ${ }^{2}$ Ischaemic stroke accounts for $69.6 \%$ of all strokes and the incidence of stroke has continued to steeply rise over the last three decades. ${ }^{3}{ }^{4}$ Although gradual improvements in stroke care have been achieved in recent years, regional differences still exist in the quality of diagnostic measures and stroke recurrence. ${ }^{5-7}$

Accurate classification of ischaemic stroke aetiology is indispensable for patient management and research because stroke prognosis ${ }^{8}$ and strategies for secondary prevention of stroke differ by stroke subtype. ${ }^{9-11}$ There are several aetiological subtyping systems ${ }^{12-19}$; however, despite the limited discriminatory ability and prognostic value, ${ }^{8}$ the Trial of Org 10172 in Acute Stroke Treatment (TOAST) system ${ }^{12}$ remains the most widely used aetiological categorising system in clinical research and practice. $^{2021}$

In clinical practice and most clinical trials, aetiological diagnosis is based on the judgement of a local neurologist or site investigator. Previous studies have reported only moderate inter-rater reliability ( $\kappa: 0.42-0.64$ ) with the TOAST system. ${ }^{22}{ }^{23}$ Both standardised medical record review ${ }^{24}$ and centralised adjudication ${ }^{25}$ have been shown to improve this situation. However, the accuracy of the routine aetiological diagnosis of ischaemic stroke according to TOAST criteria when the reported subtype is assumed to be correct has not been validated yet.

To the best of our knowledge, no large-scale study has compared the centralised versus non-centralised aetiological diagnosis of ischaemic stroke. The potential for heterogeneity in patient treatment following a centralised versus non-centralised diagnosis also warrants discussion. We, therefore, investigated the consistency between site-reported stroke subtypes at discharge according to centralised and non-centralised TOAST criteria for stroke diagnosis in the Third China National Stroke Registry (CNSR-III), its influence on aetiological evaluation during hospitalisation and its impact on secondary prevention strategy. 


\section{METHODS}

\section{Study design}

This study is an analysis of patients within the CNSR-III, a prospective patient cohort with ischaemic stroke or transient ischaemic attack (TIA) in China. Patients in our study were recruited between August 2015 and March 2018 from 201 study sites covering 26 provinces and municipalities in China. ${ }^{26}$ Detailed inclusion and exclusion criteria are described in the CNSR-III study protocol.

\section{Study population and standardised diagnoses}

The present analysis included all patients with ischaemic stroke with complete diffusion-weighted imaging (DWI) data as well as a complete aetiological evaluation and a non-centralised TOAST subtype designated by a site investigator at discharge. Site investigators and evaluators made decisions based on the 1993 version of the TOAST standard, ${ }^{12}$ according to a previously published study protocol. ${ }^{26}$ The TOAST system stratifies patients with ischaemic stroke into five subtypes: large artery atherosclerosis (LAA), cardioembolism (CE), small-vessel occlusion (SVO), stroke of other aetiology (OE) and stroke of undetermined cause (UE). A detailed description of the training process is included in the online supplemental materials (see online supplemental methods).

\section{Centralised and non-centralised subtyping}

To ensure that the diagnosis standard was consistent, we adopted the use of just one version of the TOAST subtype system $^{12}$ for the centralised decision algorithm. A detailed methodology of the centralised and non-centralised subtyping is described in the supplementary methods (see online supplemental methods, online supplemental materials-screening report form and online supplemental materials-decision algorithm).

\section{Statistical analysis}

A $\chi^{2}$ test or a Fisher's exact test was used to compare categorical variables. Continuous variables were compared using either one-way analysis of variance or the KruskalWallis test, where appropriate. The heterogeneity of centralised versus non-centralised subtype consistency was assessed using the $\chi^{2}$ test. To analyse the potential causes of differences between centralised and non-centralised subtypes, inpatient department, hospital level and geographic location were also compared. Patients were further stratified into three categories per subtype based on centralised/non-centralised subtype consistency: consistent, centrally reassigned and newly diagnosed. For each specific subtype, the centrally reassigned group was defined as the group of patients diagnosed with the subtype at discharge but reassigned to a different subtype after centralised diagnosis. The newly diagnosed group for a given subtype included patients who were initially diagnosed with a subtype at discharge and then newly diagnosed with the given subtype after centralised diagnosis. For example, a patient originally diagnosed as $\mathrm{OE}$ and then centrally reassigned as $\mathrm{CE}$ would be included in the centrally reassigned group for $\mathrm{OE}$ and the newly diagnosed group for CE. We used multivariate Cox proportional hazards regression to evaluate the factors associated with the inconsistency between centralised and noncentralised subtypes. Variables showing statistical differences in univariate analysis at baseline were modelled as confounding factors. We imputed missing values of the baseline variables used to adjust the regression models with mean or mode, as appropriate. No outcomes were imputed. In-hospital and discharge medication prescriptions were displayed for each category in every subtype. Indications of secondary prevention strategies were determined in accordance with established guidelines. ${ }^{10}{ }^{27} \mathrm{We}$ performed sensitivity analyses using only the patient subgroup with complete information, that is, having DWI imaging, $\geq 1$ extracranial artery evaluation, $\geq 1$ intracranial artery evaluation, $\geq 1$ cardiac rhythm evaluation (ECG (EKG) or 24-hour Holter) and $\geq 1$ cardiac structure evaluation (transthoracic echocardiography (TTE) or transoesophageal echocardiography).

A two-sided $p$ value $<0.05$ was considered to be statistically significant. All statistical analyses were performed using SAS V.9.4 (SAS Institute, Cary, North Carolina, USA).

\section{RESULTS}

\section{Participant distribution and baseline characteristics}

This study included a total of 12180 patients with acute ischaemic stroke after excluding 1020 patients with TIA and 1966 patients with incomplete MRI data (figure 1). The included patients were older and had a lower rate of TIA history, myocardial infarction history and atrial fibrillation history; however, a higher percentage of patients had dyslipidaemia, were current smokers and or were diagnosed with cerebral infarction (see online supplemental table I).

Baseline characteristics of the study population are listed in online supplemental table II). The average age of the study population was 62.3 years (SD: 11.2 years), and $31.4 \%$ of all the included patients were women. A total of $11586(95.1 \%)$ patients underwent intracranial artery evaluation and $11454(94.0 \%)$ patients underwent a complete extracranial artery test. TTE data were available for $94.7 \%$ of the patients, EKG data for $94.3 \%$ and Holter data for $83.6 \%$.

\section{Consistency between centralised and non-centralised subtype distributions}

Differences between the distributions of aetiological subtypes based on discharge and those based on the centralised subtyping system are presented in figure 2. Substantial differences were observed between the centralised and non-centralised subtypes. Only 4316 patients $(35.4 \%)$ were assigned to the same aetiological category after the centralised subtyping procedure performed by the site investigator at discharge. Agreement between the centralised and non-centralised subtypes was highest for 


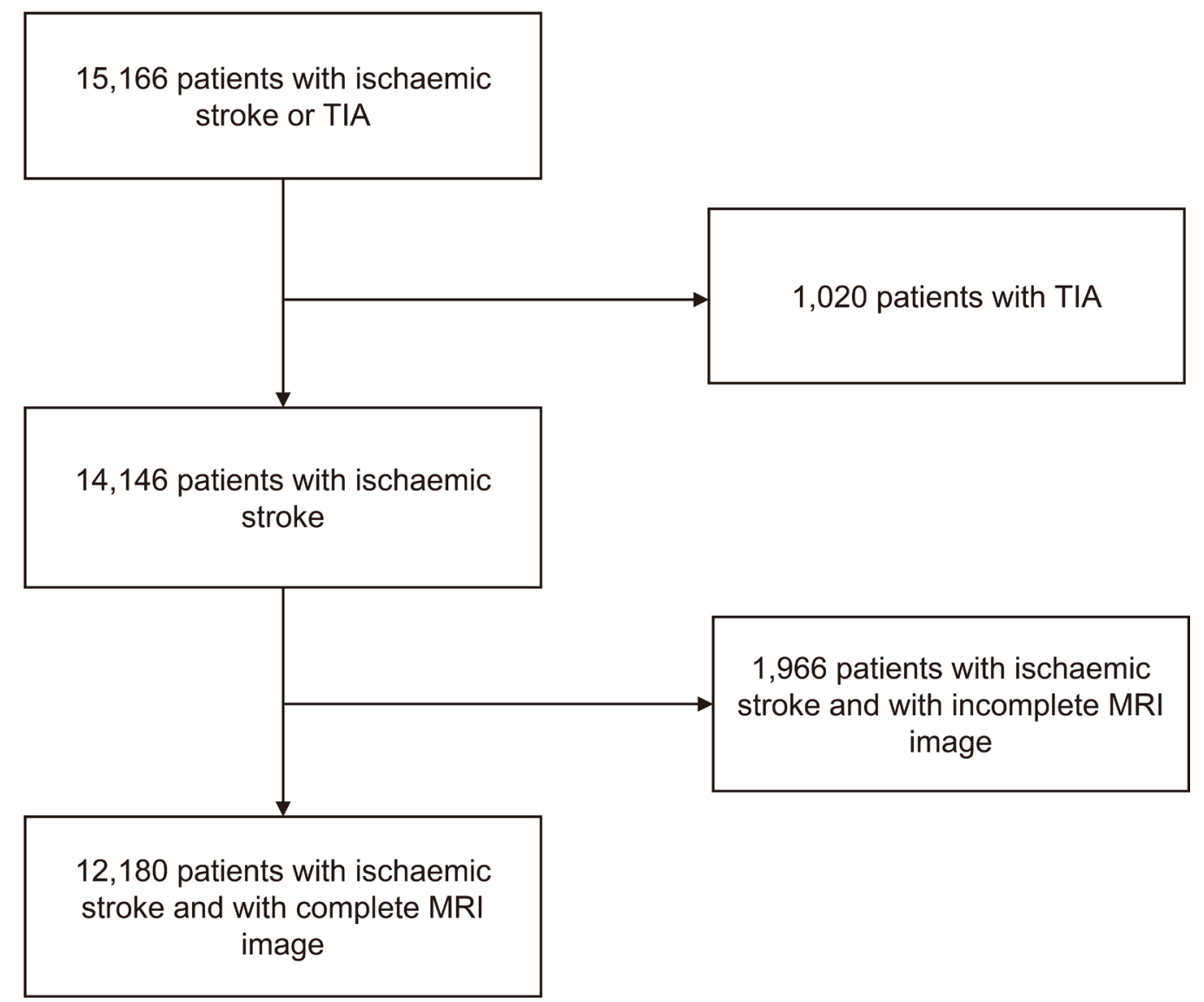

Figure 1 Flow chart of patient inclusion and exclusion criteria.

the LAA subtype $(77.4 \%$ of patients centrally diagnosed as LAA), followed by the SVO type ( $40.6 \%$ of patients centrally diagnosed as SVO). The agreement of CE and OE subtypes was $38.7 \%$ and $12.2 \%$, respectively. Similar results were observed in the subgroup of patients with complete information.

To identify the potential reasons for the inconsistency between discharge and centralised subtype diagnosis, we compared both patient-level (demographic characteristics, baseline National Institutes of Health Stroke Scale (NIHSS) and so on) and hospital level (inpatient department, hospital type and geographic location or area of the hospital) factors (see online supplemental table III).

In multivariate logistic regression analysis, a history of ischaemic stroke (adjusted OR 0.841; 95\% CI 0.749 to 0.943 ), TIA (adjusted OR $0.606 ; 95 \%$ CI 0.451 to 0.815 ) and dyslipidaemia (adjusted OR, 0.822; 95\% CI 0.689 to 0.980 ) were each associated with fewer patients who were centrally reassigned from the LAA subtype. Additionally, hospitals in the urban area (adjusted OR 0.792; 95\% CI 0.687 to 0.913 ) and tertiary hospitals (adjusted OR 0.748 ; $95 \%$ CI 0.627 to 0.891 ) were each associated with fewer patients centrally reassigned from the LAA subtype. Furthermore, we observed a significant interaction $(\mathrm{p}=0.005)$ of hospital area with hospital type in the consistency of LAA diagnosis. Non-stroke unit inpatient departments were associated with more patients being centrally reassigned from the LAA subtype (adjusted OR 1.140; $95 \%$ CI 1.012 to 1.284). Hospitals in the middle (adjusted OR 0.445 ; $95 \%$ CI 0.330 to 0.600 ) and the eastern (adjusted OR $0.476 ; 95 \%$ CI 0.359 to 0.631 ) regions of China were both associated with less newly diagnosed patients, indicating a more consistent LAA diagnosis.

A history of atrial fibrillation or flutter was associated with a more consistent diagnosis of the CE subtype (centrally reassigned CE group: adjusted OR 0.122; $95 \%$ CI 0.070 to 0.213 ; newly diagnosed CE group: adjusted OR 0.086; 95\% CI 0.053 to 0.140). Evaluation of cardiac rhythm via 24-hour Holter was related to a more consistent CE subtype diagnosis (newly diagnosed CE group: adjusted OR $0.567 ; 95 \%$ CI 0.350 to 0.919 ).

A higher baseline NIHSS score was associated with more centrally reassigned patients with SVO (adjusted OR $1.360 ; 95 \%$ CI 1.164 to 1.588 ) and more newly diagnosed patients with SVO (adjusted OR 1.452; 95\% CI 1.236 to 1.707). Additionally, tertiary hospitals were associated with more newly diagnosed patients with SVO (adjusted OR $1.410 ; 95 \%$ CI 1.140 to 1.743 ), while hospitals in urban areas were associated with fewer newly diagnosed patients with SVO (adjusted OR 0.446; 95\% CI 0.360 to 0.552 ). An interaction was observed between hospital type, location/ area and consistency of SVO diagnosis $(\mathrm{p}=0.037)$.

Older age was associated with a high proportion of being centrally reassigned from the OE subtype (adjusted 

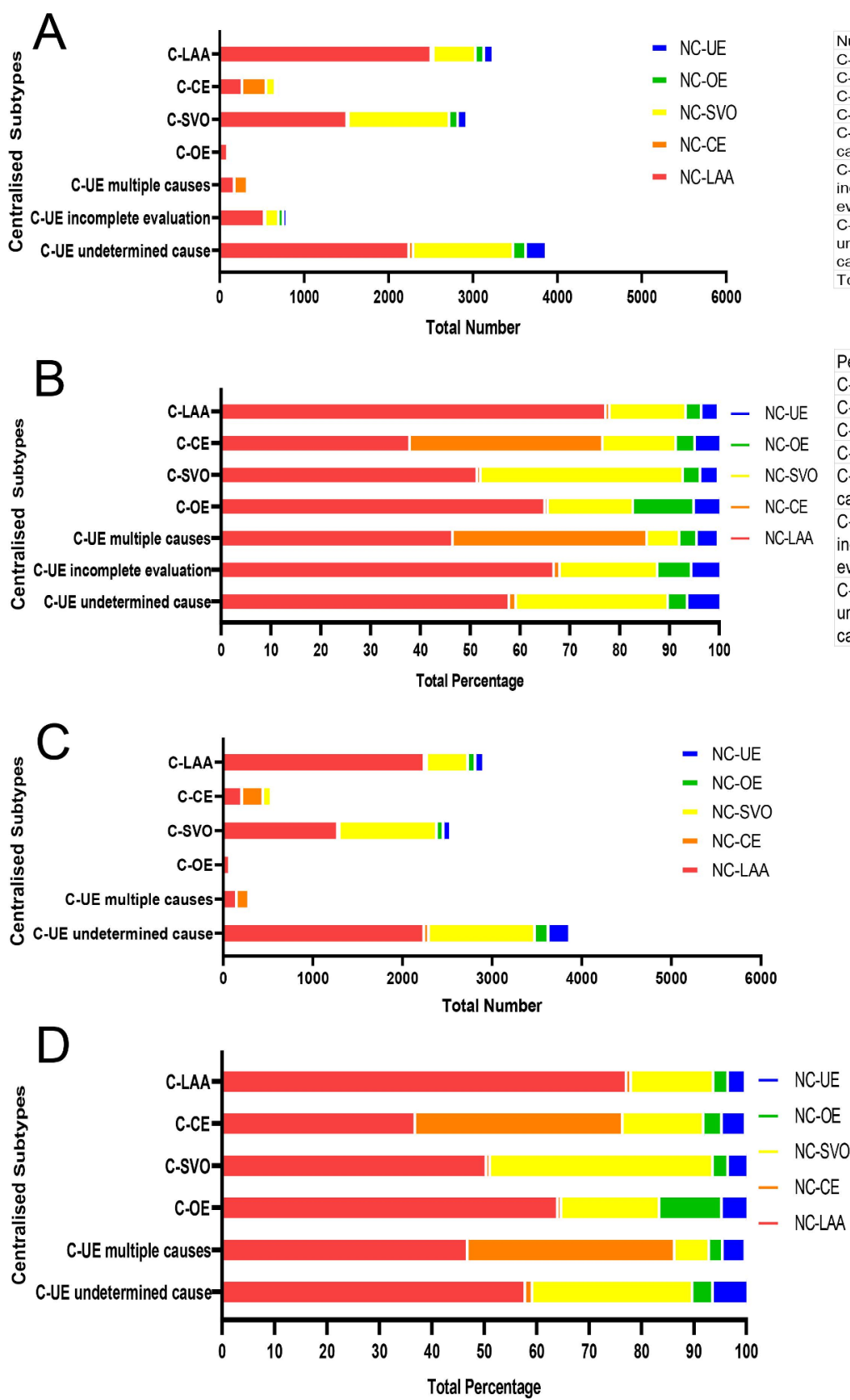

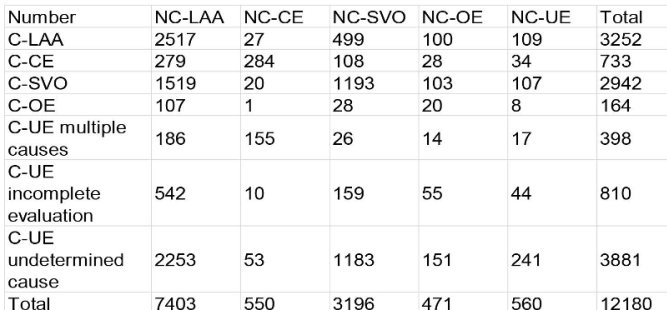

Percentage NC-LAA NC-CE NC-SVO NC-OE NC-UE Total

$\begin{array}{lllllll}\text { C-LAA } & 77.4 \% & 0.8 \% & 15.3 \% & 3.1 \% & 3.4 \% & 100.0 \%\end{array}$

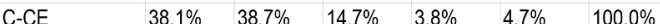

\begin{tabular}{llllllll}
\hline C-SVO & $51.6 \%$ & $0.7 \%$ & $40.6 \%$ & $3.5 \%$ & $3.6 \%$ & $100.0 \%$
\end{tabular}

$\begin{array}{lllllll}\text { C-OE } & 65.2 \% & 0.6 \% & 17.1 \% & 12.2 \% & 4.9 \% & 100.0 \%\end{array}$

$\begin{array}{lllllll}\text { C-UE multiple } & 46.7 \% & 39.0 \% & 6.5 \% & 3.5 \% & 4.3 \% & 100.0 \%\end{array}$

Causes

$\begin{array}{lllllll}\text { incomplete } & 67.0 \% & 1.2 \% & 19.6 \% & 6.8 \% & 5.4 \% & 100.0 \%\end{array}$

evaluation

C-UE

$\begin{array}{lllllll}\text { undetermined } & 58.0 \% & 1.4 \% & 30.5 \% & 3.9 \% & 6.2 \% & 100.0 \%\end{array}$

cause

Figure 2 Centralised aetiological subtype and individualised subtype distribution. (A) Total number of each subtype in the study population. (B) Distribution of non-centralised subtyping results according to the percentages of centralised subtypes in the study population. (C) Total number of each subtype in the subgroup with complete information. (D) Distribution of non-centralised subtyping results according to the percentages of centralised subtypes in the subgroup with complete information. C, centralised; CE, cardioembolism; NC, non-centralised; OE, other determined cause; SVO, small-vessel occlusion; UE-multiple causes, with concomitant causes; UE-incomplete evaluation; UE-undetermined cause; LAA, large-artery atherosclerosis.

OR 1.062; 95\% CI 1.022 to 1.102 ) and being newly diagnosed with OE (adjusted OR 1.062; 95\% CI 1.016 to 1.109). Several patient-level and hospital-level factors were associated with agreement between centralised and non-centralised aetiological subtyping (table 1).

Aetiological diagnosis and patient management

Fewer patients with newly diagnosed CE underwent 24-hour Holter $(82.1 \%)$ than the patients in both the consistent $\mathrm{CE}(87.8 \%)$ and centrally reassigned $\mathrm{CE}$ groups $(87.7 \%$; see online supplemental table III). The prescription rate of oral anticoagulants for secondary prevention was lower in newly diagnosed patients with CE $(10 \%$ vs $50.2 \%$ for the consistent CE group and $27.6 \%$ for the centrally reassigned CE group, $\mathrm{p}<0.001$ ). The prescription rate of antihypertensive agents was the highest in the consistent SVO group (48.7\% vs $42.7 \%$ for the centrally reassigned SVO group and $44.3 \%$ for the newly diagnosed SVO group). Prescription rates 
Table 1 Multivariate analysis of potential causes of inconsistency between individualised aetiological classification and centralised classification of the study population

\begin{tabular}{|c|c|c|c|c|c|}
\hline \multicolumn{2}{|l|}{ Characteristics } & \multicolumn{2}{|l|}{ Centrally reassigned LAA } & \multicolumn{2}{|l|}{ Newly diagnosed LAA } \\
\hline Variables & $\begin{array}{l}\text { Consistent LAA as } \\
\text { reference }\end{array}$ & Adjusted OR $(95 \% \mathrm{Cl})$ & $P$ value & Adjusted OR (95\% Cl) & $P$ value \\
\hline \multicolumn{6}{|l|}{ Medical history } \\
\hline Ischaemic stroke & Yes & 0.841 (0.749 to 0.943 ) & 0.003 & 0.843 (0.687 to 1.035$)$ & 0.103 \\
\hline TIA & Yes & 0.606 (0.451 to 0.815$)$ & $<0.001$ & 0.585 (0.333 to 1.028$)$ & 0.062 \\
\hline Dyslipidaemia & Yes & 0.822 (0.689 to 0.980$)$ & 0.029 & 0.806 (0.587 to 1.106$)$ & 0.181 \\
\hline \multirow[t]{2}{*}{ Baseline NIHSS score } & $0-3$ & 1.000 (ref) & Ref & 1.000 (ref) & Ref \\
\hline & $\geq 4$ & 0.731 (0.663 to 0.806$)$ & $<0.0001$ & 0.537 (0.453 to 0.636$)$ & $<0.0001$ \\
\hline \multirow[t]{2}{*}{ Inpatient department } & Stroke unit & 1.000 (ref) & Ref & 1.000 (ref) & Ref \\
\hline & Other & $1.140(1.012$ to 1.284$)$ & 0.031 & $1.037(0.843$ to 1.274$)$ & 0.733 \\
\hline \multirow[t]{3}{*}{ Geographical region } & West & 1.000 (ref) & Ref & 1.000 (ref) & Ref \\
\hline & Middle & 0.941 (0.770 to 1.150$)$ & 0.551 & 0.445 (0.330 to 0.600$)$ & $<0.0001$ \\
\hline & East & 0.882 (0.726 to 1.072$)$ & 0.207 & 0.476 (0.359 to 0.631$)$ & $<0.0001$ \\
\hline \multirow[t]{2}{*}{ Area } & Rural & 1.000 (ref) & Ref & 1.000 (ref) & Ref \\
\hline & Urban & 0.792 (0.687 to 0.913$)$ & 0.001 & 1.163 (0.911 to 1.484$)$ & 0.226 \\
\hline \multirow[t]{2}{*}{ Hospital type } & Secondary & 1.000 (ref) & Ref & 1.000 (ref) & Ref \\
\hline & Tertiary & 0.748 (0.627 to 0.891$)$ & 0.001 & 0.796 (0.591 to 1.072$)$ & 0.133 \\
\hline \multicolumn{2}{|c|}{$\mathrm{P}$ value of interaction for area and hospital type } & & 0.005 & & 0.214 \\
\hline \multirow[t]{2}{*}{ Variables } & \multirow{2}{*}{$\begin{array}{l}\text { Consistent CE as } \\
\text { reference }\end{array}$} & Centrally reassigned CE & & Newly diagnosed CE & \\
\hline & & Adjusted OR (95\% Cl) & $P$ value & Adjusted OR (95\% Cl) & $P$ value \\
\hline \multicolumn{6}{|l|}{ Medical history } \\
\hline $\begin{array}{l}\text { Known atrial fibrillation or } \\
\text { flutter }\end{array}$ & Yes & $0.122(0.070$ to 0.213$)$ & $<0.0001$ & $0.086(0.053$ to 0.140$)$ & $<0.0001$ \\
\hline Diabetes mellitus & Yes & 1.015 (0.599 to 1.721$)$ & 0.955 & 1.667 (1.060 to 2.623$)$ & 0.027 \\
\hline \multirow[t]{2}{*}{ Baseline NIHSS score } & $0-3$ & 1.000 (ref) & Ref & 1.000 (ref) & Ref \\
\hline & $\geq 4$ & 1.658 (1.131 to 2.432$)$ & 0.010 & $0.759(0.541$ to 1.065$)$ & 0.110 \\
\hline \multicolumn{6}{|l|}{ Evaluation of cardiac rhythm } \\
\hline Holter & Yes & 1.077 (0.606 to 1.915$)$ & 0.800 & 0.567 (0.350 to 0.919$)$ & 0.021 \\
\hline \multirow[t]{2}{*}{ Variables } & \multirow{2}{*}{$\begin{array}{l}\text { Consistent SVO as } \\
\text { reference }\end{array}$} & \multicolumn{2}{|l|}{ Centrally reassigned SVO } & \multicolumn{2}{|l|}{ Newly diagnosed SVO } \\
\hline & & Adjusted OR (95\% Cl) & $P$ value & Adjusted OR (95\% Cl) & $P$ value \\
\hline Age & & $1.010(1.003$ to 1.018$)$ & 0.004 & $1.011(1.003$ to 1.019$)$ & 0.005 \\
\hline Sex & & 0.970 (0.813 to 1.158$)$ & 0.738 & 0.741 (0.613 to 0.896$)$ & 0.002 \\
\hline mRS prior to current event & & 1.072 (0.966 to 1.191$)$ & 0.192 & 1.215 (1.091 to 1.354$)$ & $<0.001$ \\
\hline \multicolumn{6}{|l|}{ Medical history } \\
\hline Current or previous smoker & Yes & 0.780 (0.655 to 0.929$)$ & 0.005 & 0.858 (0.716 to 1.028$)$ & 0.096 \\
\hline \multirow[t]{2}{*}{ Baseline NIHSS score } & $0-3$ & 1.000 (ref) & Ref & 1.000 (ref) & Ref \\
\hline & $\geq 4$ & 1.360 (1.164 to 1.588$)$ & 0.0001 & 1.452 (1.236 to 1.707$)$ & $<0.0001$ \\
\hline \multirow[t]{3}{*}{ Geographical region } & West & 1.000 (ref) & Ref & 1.000 (ref) & Ref \\
\hline & Middle & 1.320 (0.982 to 1.775$)$ & 0.066 & 1.447 (1.079 to 1.940$)$ & 0.014 \\
\hline & East & 1.383 (1.039 to 1.839$)$ & 0.026 & 1.125 (0.849 to 1.490$)$ & 0.412 \\
\hline \multirow[t]{2}{*}{ Area } & Rural & 1.000 (ref) & Ref & 1.000 (ref) & Ref \\
\hline & Urban & 0.875 (0.713 to 1.074$)$ & 0.201 & $0.446(0.360$ to 0.552$)$ & $<0.0001$ \\
\hline \multirow[t]{2}{*}{ Hospital type } & Secondary & 1.000 (ref) & Ref & 1.000 (ref) & Ref \\
\hline & Tertiary & 0.839 (0.662 to 1.064$)$ & 0.148 & $1.410(1.140$ to 1.743$)$ & 0.002 \\
\hline
\end{tabular}


Table 1 Continued

\begin{tabular}{|c|c|c|c|c|c|}
\hline \multicolumn{2}{|l|}{ Characteristics } & \multicolumn{2}{|l|}{ Centrally reassigned LAA } & \multicolumn{2}{|l|}{ Newly diagnosed LAA } \\
\hline Variables & $\begin{array}{l}\text { Consistent LAA as } \\
\text { reference }\end{array}$ & Adjusted OR (95\% Cl) & $P$ value & Adjusted OR (95\% Cl) & $P$ value \\
\hline Variables & $\begin{array}{l}\text { Consistent OE as } \\
\text { reference }\end{array}$ & \multicolumn{2}{|l|}{ Centrally reassigned OE } & \multicolumn{2}{|l|}{ Newly diagnosed OE } \\
\hline Sex & & $0.293(0.112$ to 0.765$)$ & 0.012 & 0.701 (0.245 to 2.005$)$ & 0.507 \\
\hline \multicolumn{6}{|l|}{ Evaluation of cardiac rhythm } \\
\hline EKG & Yes & $1.702(0.487$ to 5.947$)$ & 0.405 & $\begin{array}{l}10.173 \text { (2.019 to } \\
51.258)\end{array}$ & 0.005 \\
\hline
\end{tabular}

West includes Inner Mongolia, Guangxi, Chongqing, Sichuan,Guizhou, Yunnan, Tibet, Shaanxi, Gansu, Qinghai, Ningxia and Xinjiang. Middle includes Shanxi, Anhui, Jiangxi, Henan, Hubei and Hunan. East includes Beijing, Tianjin, Hebei, Shanghai, Jiangsu, Zhejiang, Fujian,Shandong, Guangdong, Hainan, Liaoning, Jilin and Heilongjian.

CE, cardioembolism; LAA, large artery atherosclerosis; mRS, modified Rankin Scale; NIHSS, National Institutes of Health Stroke Scale; OE, other determined cause; ref, reference; SVO, small vessel occlusion.

for guideline-recommended treatments other than antithrombotic agents (such as lipid-lowering, antidiabetic and antihypertensive treatments) were comparable among the different groups based on the consistency of centralised and non-centralised subtyping in patients with LAA (table 2). Similar results were observed in the patient subgroup with complete information (see online supplemental table IV).

Of the 449 newly diagnosed patients with CE stroke, $431(95.9 \%)$ had high-risk sources of CE. Among the high-risk sources, the largest proportion was newly diagnosed atrial fibrillation $(58.47 \%)$, followed by a history of atrial fibrillation $(28.54 \%$; figure 3$)$.

Oral anticoagulant prescription rates were significantly different among the three groups of patients with $\mathrm{CE}$ categorised by agreement between centralised and noncentralised subtyping. Among patients with indications for anticoagulation therapy, the prescription rate of oral anticoagulants at discharge in the newly diagnosed CE group was surprisingly lower $(15.3 \%)$ than that in the consistent CE (51.3\%) and the centrally reassigned CE groups (42.0\%; table 3$)$. Similar results were also observed in the patient subgroup with complete information (see online supplemental table V).

\section{DISCUSSION}

In the current study, we found substantial differences between centralised and non-centralised aetiological classifications of stroke subtypes in China. Factors related to the heterogeneity between centralised and non-centralised subtyping included the hospital level, geographical region/location and area of the admitting hospital; the admitting department and stroke severity. Incomplete aetiological investigation during hospitalisation and inadequate secondary prevention strategies were observed in patients with inconsistent classification by site evaluators (non-centralised) using centralised adjudication. Furthermore, we found that both a high baseline NIHSS score and admission to a stroke unit were each associated with a more consistent aetiological diagnosis between centralised and non-centralised stroke subtyping. Thus, differences in evaluation and care quality among patients with different severities might lead to inaccurate aetiological diagnoses in patients with mild neurological deficits.

The proportion of distribution of each subtype after centralised and non-centralised TOAST classification was similar to those reported in a previous registry, ${ }^{28}$ with LAA accounting for a large proportion of ischaemic stroke and $\mathrm{CE}$ accounting for a small proportion. The particularly high prevalence of intracranial artery stenosis in Chinese patients with stroke ${ }^{29}$ might explain the high-ranking proportion of the LAA subtype relative to the other subtypes. However, the proportion of LAA subtype among all patients classified by the non-centralised or centralised designations was different $(60.8 \%$ vs $26.7 \%)$. A strict application of the TOAST classification criteria can lead to the designation of a significant number of strokes as an undetermined cause category. ${ }^{14}$ A neurologist's 'clinical opinion', based on experience, might help to assign a high degree of confidence to one specific stroke cause in a particular patient when facing competing evidence of different aetiologies. Recent subtyping systems, such as the Chinese Ischaemic Stroke Subclassification (CISS) criteria, emphasise underlying pathological mechanisms and contain revised standards for categorising the LAA subtype. ${ }^{19}$ According to CISS criteria, patients with infarct in the territory of an isolated penetrating artery and evidence of atherosclerotic plaque or any degree of stenosis in the parent artery are categorised into the LAA subtype, regardless of the degree of luminal stenosis of the clinically relevant artery. With the continuously 


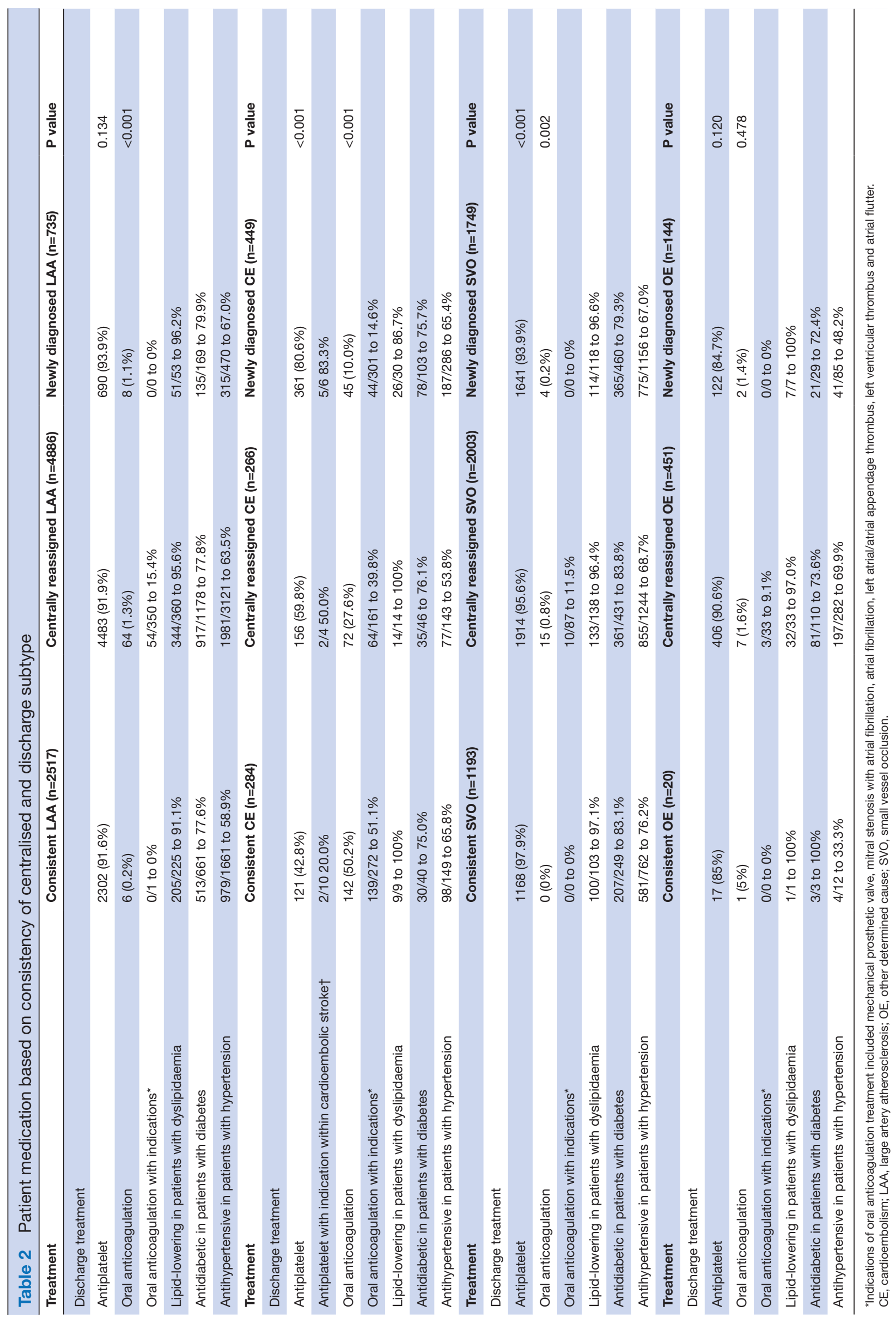




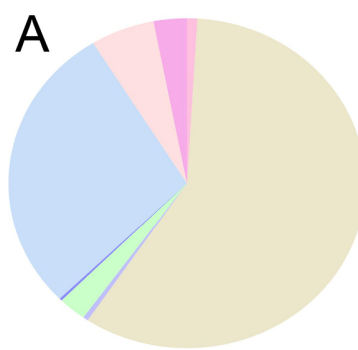

$0.93 \%$ Mechanical prosthetic valve

$58.47 \%$ Newly diagnosed atrial fibrillation

$0.46 \%$ Left atrial/atrial appendage thrombus

$2.55 \%$ Recent myocardial infarction ( $<4$ weeks)

$0.23 \%$ Atrial myxoma

$28.54 \%$ History of atrial fibrillation

$5.80 \%$ Dialated cardiomyopathy diagnosed by echocardiograph

$3.02 \%$ Myocardial infarction ( $>4$ weeks, $<6$

months)

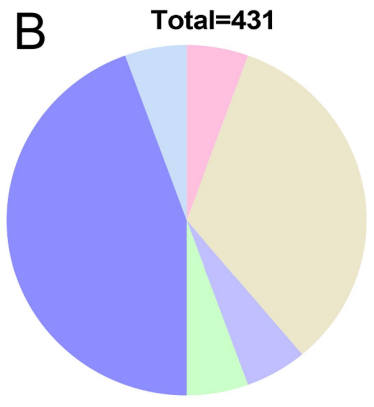

$5.56 \%$ Mitral valve prolapse

$58.47 \%$ Newly diagnosed atrial fibrillation

$5.56 \%$ Mitral stenosis without atrial fibrillation

$5.56 \%$ Patent foramen ovale

- $44.44 \%$ Atrial flutter

-5.56\% Hypokinetic left ventricular segment

Total $=18$

Figure 3 Proportions of high-risk and medium-risk embolic sources for newly diagnosed patients with cardioembolic stroke.

Panel A: proportions of high-risk sources. Panel B: proportions of medium-risk sources.

increasing development of novel techniques for plaque detection and vessel wall imaging, mild intracranial artery stenosis $(<50 \%$ luminal stenosis) or non-stenotic atherosclerotic plaques are being recognised in $>50 \%$ of patients with ischaemic symptoms. ${ }^{30}$ These new subtyping criteria have had a profound influence on Chinese neurologists and might bias their 'clinical opinions' towards the LAA subtype.

Unlike the considerably high incidence of the $\mathrm{CE}$ subtype in the Caucasian population, ${ }^{31}$ CE stroke accounts for only $5.7 \%$ of ischaemic strokes among Chinese patients, even with a complete aetiological investigation. Disagreements between non-centralised and centralised aetiological diagnosis of the CE subtype were mainly caused by the detection of atrial fibrillation (AF) after ischaemic stroke. AF is a well-established predictor of stroke recurrence and has a validated association with cardiogenic embolism. ${ }^{32}$ The AF-SCREEN International Collaboration recommends 72-hour electrocardiographic monitoring for AF detection. ${ }^{33}$ Because the CNSR-III protocol was written before publication of the white paper, ${ }^{33}$ a stepwise approach was used to evaluate AF using resting EKG followed by 24-hour Holter monitoring. The current diagnostic approach detected AF-related stroke in 252 patients and reassigned them to the CE category. Another reason for subtyping inconsistency might be the availability of complementary information from the centralised data collection. Newly observed high-risk or medium-risk cardiac embolism sources within centralised data contributed to $62.3 \%$ of cardioembolic stroke classifications.

Inconsistency between non-centralised and centralised classifications of the CE subtype was associated with inadequate guideline-recommended secondary prevention strategy, especially with regard to indications for treatment with oral anticoagulants. Among patients with ischaemic stroke with non-valvular atrial fibrillation (NVAF) in the CNSR without contraindications to anticoagulation, only $16.2 \%$ received warfarin therapy as secondary prevention. ${ }^{34}$ In the second CNSR (CNSR-II), the prescription rate of warfarin for patients with stroke with NVAF remained low (19.4\%). ${ }^{35}$ Although an overall increase was observed in the use of oral anticoagulants in the current analysis, we found that the prescription rate in patients with unrecognised $\mathrm{CE}$ with indications of anticoagulation treatment $(15.3 \%)$ was even lower than that reported in previous studies. ${ }^{345}$ Inaccurate diagnosis of CE aetiology could cause suboptimal anticoagulation treatment in patients with CE indicated for anticoagulant therapy. Enhancing the accuracy and reliability of aetiological diagnosis is an essential step to improve adherence to guideline-recommended secondary prevention treatment.

Our study has several strengths. We used an algorithm for centralised adjudication of aetiology categories, which integrated all necessary data elements collected and processed centrally by trained specialists. Furthermore, this study was based on a cohort in which $>94 \%$ of all included patients underwent complete aetiological examinations. The centralised aetiology classification was reproducible and stable because it eliminated any disagreements between evaluators in the judgement step.

However, this study had certain limitations. First, the algorithm was designed based on previously published rules. ${ }^{12}$ New perspectives to stratify the sources of cardiac embolism risk are continually being updated. ${ }^{36}$ 


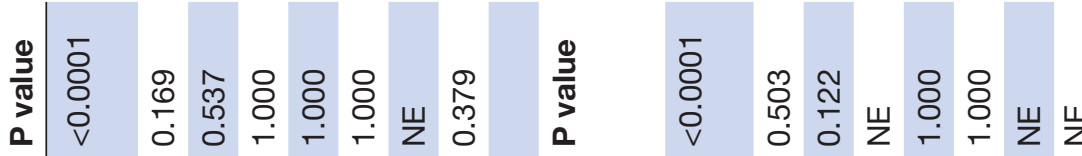

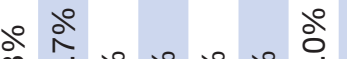

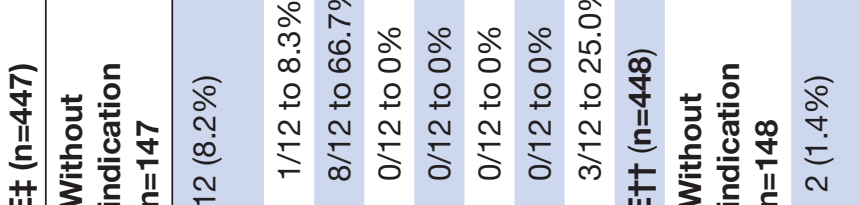

○े ๖

○े ८ั

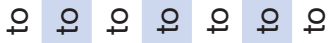

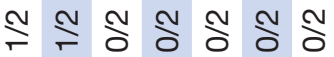

䓀

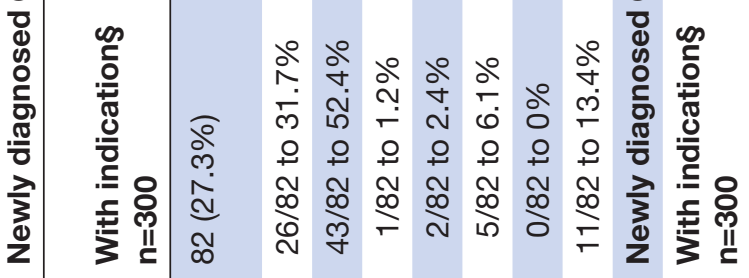

ำ

र.

우오 우 우 우 우

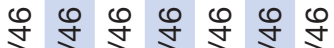

ল্লি ন ন

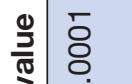

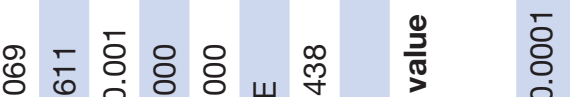

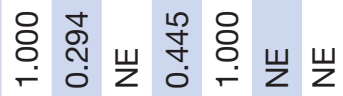

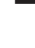

O

흘

$\frac{\sqrt{2}}{\frac{2}{2}}$

సิ

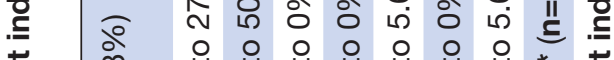

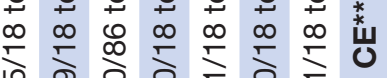

m

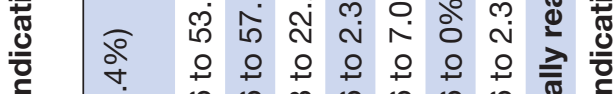

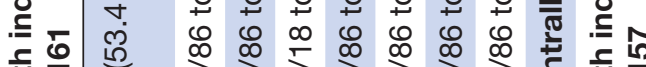

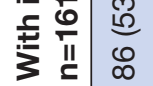

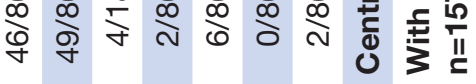

ㅇำ

ñ.

a

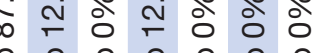

웅ㅇ 웅

$\stackrel{\infty}{\sim} \stackrel{\infty}{\sim} \stackrel{\infty}{\circ} \stackrel{\infty}{\sim} \stackrel{\infty}{\circ} \stackrel{\infty}{\circ} \stackrel{\infty}{\circ}$

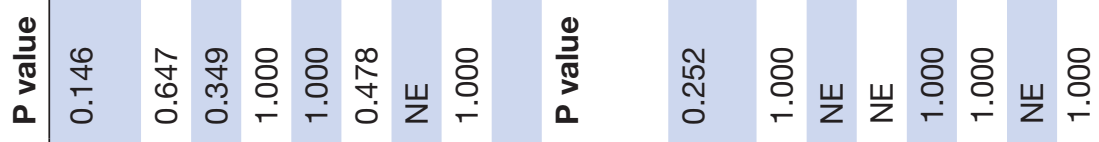

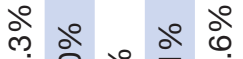

ळ

웅ㅇ 웅ㅇㅇㅇ

$\& \& \& \& \& \& \&$

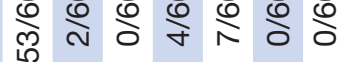

×

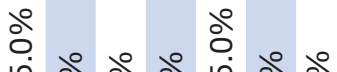

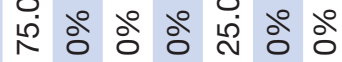
웅ㅇㅇㅇㅇㅇ 우

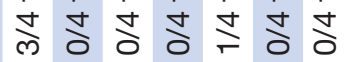


Efficient treatments for different potential embolic sources continue to be developed, such as percutaneous patent foramen ovale (PFO) closure for PFO-related stroke. ${ }^{37-39}$ For future use, the algorithm employed in the current study needs to be updated with the most recent knowledge. Second, many patients in the current study were assigned to the undetermined causes category with $\geq 1$ competing mechanism after completing the diagnostic workup. It is thus essential to refine the hierarchical competing standard among subtype elements. Third, the standard evaluation process in the current study lacked cardiac MRI, 72-hour EKG monitoring and specific biomarkers for cardiac function. Fourth, the evaluators at each study site were trained in the use of videotape. The intra-rater and inter-rater reliabilities were not centrally assessed before aetiological diagnosis was performed. However, this reflects a real-world challenge of evaluating interobserver and intraobserver agreements of aetiological diagnoses owing to the large scale of neurological departments and constantly rotating physicians in the many hospitals in China. Efforts to improve the quality of centralised training processes and constant interactive feedback are needed to narrow the gap between noncentralised and centralised subtyping in future multicentre studies.

\section{CONCLUSIONS}

We report substantial differences between discharge and centralised diagnosis of stroke aetiology. The factor most associated with these differences was the inhomogeneity among admitting departments and hospitals in their ability to identify potential stroke mechanisms. An inadequate secondary prevention strategy was observed in patients with inconsistent classification by site evaluators (non-centralised) and centralised adjudicators.

\section{Twitter Yilong Wang @yilong}

Contributors Y-JW had full access to all of the data in the study and takes responsibility for the integrity of the data and the accuracy of the data analysis. Y-JW, JJ and YS designed the protocol and framework of this study. YS, JJ, XM, YJ and YP collected the data. Huan Liu and HY conducted statistical analysis. YS drafted the manuscript. ZL, YJ, XY, LL, XZ, Y-LW and Hao Li reviewed, edited and approved the final version.

Funding This study was funded by National Science and Technology Major Project National Key R\&D Program of China Beijing Municipal Science \& Technology Commission (D171100003017002).

Competing interests None declared.

Patient consent for publication Not required.

Provenance and peer review Not commissioned; externally peer reviewed.

Data availability statement Data are available on reasonable request.

Open access This is an open access article distributed in accordance with the Creative Commons Attribution Non Commercial (CC BY-NC 4.0) license, which permits others to distribute, remix, adapt, build upon this work non-commercially, and license their derivative works on different terms, provided the original work is properly cited, appropriate credit is given, any changes made indicated, and the use is non-commercial. See: http://creativecommons.org/licenses/by-nc/4.0/.

\section{ORCID iDs}

Yue Suo http://orcid.org/0000-0002-0632-0262

Zixiao Li http://orcid.org/0000-0002-4713-5418

Yuesong Pan http://orcid.org/0000-0003-3082-6789
Liping Liu http://orcid.org/0000-0003-2943-055X

Hao Li http://orcid.org/0000-0002-8591-4105

Yongjun Wang http://orcid.org/0000-0002-9976-2341

\section{REFERENCES}

1 GBD 2016 Stroke Collaborators. Global, regional, and national burden of stroke, 1990-2016: a systematic analysis for the global burden of disease study 2016. Lancet Neurol 2019;18:439-58.

2 Zhou M, Wang $\mathrm{H}$, Zeng $\mathrm{X}$, et al. Mortality, morbidity, and risk factors in China and its provinces, 1990-2017: a systematic analysis for the global burden of disease study 2017. Lancet 2019;394:1145-58.

3 Wang W, Jiang B, Sun $\mathrm{H}$, et al. Prevalence, Incidence, and Mortality of Stroke in China: Results from a Nationwide Population-Based Survey of 480687 Adults. Circulation 2017;135:759-71.

4 Global Burden of Disease Collaborative Network. Global burden of disease study 2017 (GBD 2017) results, 2018. Available: http://ghdx. healthdata.org/gbd-results-tool [Accessed Aug 2020].

5 Gu H-Q, Yang X, Rao Z-Z, et al. Disparities in outcomes associated with rural-urban insurance status in China among inpatient women with stroke: a registry-based cohort study. Ann Transl Med 2019;7:426.

$6 \mathrm{Li} \mathrm{Z}$, Jiang Y, Li H, et al. China's response to the rising stroke burden. BMJ 2019;364:I879.

7 Wang Y, Li Z, Wang Y, et al. Chinese stroke center alliance: a national effort to improve healthcare quality for acute stroke and transient ischaemic attack: rationale, design and preliminary findings. Stroke Vasc Neurol 2018;3:256-62.

8 Arsava EM, Helenius J, Avery R, et al. Assessment of the predictive validity of etiologic stroke classification. JAMA Neurol 2017;74:419-26.

9 Powers WJ, Rabinstein AA, Ackerson T, et al. Guidelines for the early management of patients with acute ischemic stroke: a guideline for healthcare professionals from the American heart Association/ American stroke association. Stroke 2018;2018:e46-110.

10 Kernan WN, Ovbiagele B, Black HR, et al. Guidelines for the prevention of stroke in patients with stroke and transient ischemic attack: a guideline for healthcare professionals from the American heart Association/American stroke association. Stroke 2014;45:2160-236.

11 National Collaborating Centre for Chronic Conditions. National clinical guideline for diagnosis and initial management of acute stroke and transient ischaemic attack (TIA. London, United Kingdom: Royal College of Physicians of London, 2008.

12 Adams HP, Bendixen BH, Kappelle LJ, et al. Classification of subtype of acute ischemic stroke. definitions for use in a multicenter clinical trial. TOAST. trial of ORG 10172 in acute stroke treatment. Stroke 1993;24:35-41

13 Ay $\mathrm{H}$, Benner T, Arsava EM, et al. A computerized algorithm for etiologic classification of ischemic stroke: the causative classification of stroke system. Stroke 2007;38:2979-84.

14 Ay H, Furie KL, Singhal A, et al. An evidence-based causative classification system for acute ischemic stroke. Ann Neurol 2005;58:688-97.

15 Arsava EM, Ballabio E, Benner T, et al. The causative classification of stroke system: an international reliability and optimization study. Neurology 2010;75:1277-84.

16 Amarenco P, Bogousslavsky J, Caplan LR, et al. New approach to stroke subtyping: the A-S-C-O (phenotypic) classification of stroke. Cerebrovasc Dis 2009;27:502-8.

17 Amarenco P, Bogousslavsky J, Caplan LR, et al. The ASCOD phenotyping of ischemic stroke (updated ASCO phenotyping). Cerebrovasc Dis 2013;36:1-5.

18 Chen P-H, Gao S, Wang Y-J, et al. Classifying ischemic stroke, from TOAST to CISS. CNS Neurosci Ther 2012;18:452-6.

19 Gao S, Wang YJ, Xu AD, et al. Chinese ischemic stroke subclassification. Front Neurol 2011;2:6.

20 Perera KS, KKH N, Nayar S, et al. Association between lowdose rivaroxaban with or without aspirin and ischemic stroke subtypes: a secondary analysis of the COMPASS trial. JAMA neurol 2019;77:43-8.

21 Mazzucco S, Li L, Binney L, et al. Prevalence of patent foramen ovale in cryptogenic transient ischaemic attack and non-disabling stroke at older ages: a population-based study, systematic review, and metaanalysis. Lancet Neurol 2018;17:609-17.

22 Atiya M, Kurth T, Berger K, et al. Interobserver agreement in the classification of stroke in the women's health study. Stroke 2003;34:565-7.

23 Gordon DL, Bendixen BH, Adams HP, et al. Interphysician agreement in the diagnosis of subtypes of acute ischemic stroke: 
implications for clinical trials. The TOAST Investigators. Neurology 1993:43:1021-7.

24 Goldstein LB, Jones MR, Matchar DB, et al. Improving the reliability of stroke subgroup classification using the trial of ORG 10172 in acute stroke treatment (TOAST) criteria. Stroke 2001;32:1091-7.

25 Majersik JJ, Cole JW, Golledge J, et al. Recommendations from the International stroke genetics Consortium, part 1: standardized phenotypic data collection. Stroke 2015;46:279-84.

26 Wang Y, Jing J, Meng X, et al. The third China national stroke registry (CNSR-III) for patients with acute ischaemic stroke or transient ischaemic attack: design, rationale and baseline patient characteristics. Stroke Vasc Neurol 2019;4:158-64.

27 Liu L, Chen W, Zhou H, et al. Chinese stroke association guidelines for clinical management of cerebrovascular disorders: Executive summary and 2019 update of clinical management of ischaemic cerebrovascular diseases. Stroke Vasc Neurol 2020;5:159-76.

28 Wang $\mathrm{Y}, \mathrm{Xu}$ J, Zhao X, et al. Association of hypertension with stroke recurrence depends on ischemic stroke subtype. Stroke 2013;44:1232-7.

29 Wang Y, Zhao X, Liu L, et al. Prevalence and outcomes of symptomatic intracranial large artery stenoses and occlusions in China: the Chinese intracranial atherosclerosis (CICAS) study. Stroke 2014:45:663-9.

30 Wang Y, Liu X, Wu X, et al. Culprit intracranial plaque without substantial stenosis in acute ischemic stroke on vessel wall MRI: a systematic review. Atherosclerosis 2019;287:112-21.

31 Ornello R, Degan D, Tiseo C, et al. Distribution and temporal trends from 1993 to 2015 of ischemic stroke subtypes: a systematic review and meta-analysis. Stroke 2018;49:814-9.
32 Lip GYH, Hunter TD, Quiroz ME, et al. Atrial fibrillation diagnosis timing, ambulatory ECG monitoring utilization, and risk of recurrent stroke. Circ Cardiovasc Qual Outcomes 2017;10:e002864.

33 Schnabel RB, Haeusler KG, Healey JS, et al. Searching for atrial fibrillation poststroke: a white paper of the AF-SCREEN international collaboration. Circulation 2019;140:1834-50.

34 Wang C, Yang Z, Wang C, et al. Significant underuse of warfarin in patients with nonvalvular atrial fibrillation: results from the China national stroke Registry. J Stroke Cerebrovasc Dis 2014;23:1157-63.

35 Yang X, Li Z, Zhao X, et al. Use of warfarin at discharge among acute ischemic stroke patients with nonvalvular atrial fibrillation in China. Stroke 2016;47:464-70.

36 Pepi M, Evangelista A, Nihoyannopoulos P, et al. Recommendations for echocardiography use in the diagnosis and management of cardiac sources of embolism: European association of echocardiography (EAE) (a registered branch of the ESC). Eur $J$ Echocardiogr 2010;11:461-76.

37 Saver JL, Carroll JD, Thaler DE, et al. Long-Term outcomes of patent foramen ovale closure or medical therapy after stroke. $N$ Engl J Med 2017;377:1022-32.

38 Mas J-L, Derumeaux G, Guillon B, et al. Patent foramen ovale closure or anticoagulation vs. antiplatelets after stroke. N Engl J Med 2017;377:1011-21.

39 Søndergaard L, Kasner SE, Rhodes JF, et al. Patent foramen ovale closure or antiplatelet therapy for cryptogenic stroke. N Engl J Med 2017;377:1033-42. 